\title{
A DYNAMIC METHOD FOR DETERMINING THE CHARACTERISTICS OF THREE-ELECTRODE VACUUM TUBES*
}

\author{
By \\ JoHN M. Miller \\ (Bureau of Standards, Washington, D. C.)
}

In three-electrode vacuum tubes, such as the audion or pliotron, we are concerned with two circuits, that between the grid and filament or input circuit and that between the plate and filament or output circuit. The current which flows in the grid circuit is of importance in determining the power input and the detecting action of the tube, but in the use of the tube as a relay or amplifier it is usually negligible and will not be considered in the following.

Characteristic Surface and Curves of the Plate CurRENT

In tubes, with a high vacuum, the value of the plate current, at a given instant, is a function of the values of the plate and grid voltages at the same instant. A surface, called the characteristic surface, is required to represent this function. Langmuir ${ }^{1}$ has given the equation

$$
i_{p}=A\left(v_{p}+k v_{g}\right)^{3 / 2}
$$

as representing the equation of the characteristic surface. In this equation $i_{p}$ is the plate current, $v_{p}$ the plate voltage, $v_{\theta}$ the grid voltage, and $k$ is a constant for a given tube construction and is a relative measure of the effects of grid and plate voltages upon the plate current.

In investigating the functioning of the tubes experimentally, it is customary to determine the static characteristic curves of the plate current. These curves are the intersections of the characteristic surface with the plane surfaces $v_{p}=$ constant, or $v_{g}=$ constant. The first of these represents the variation of the plate current with the grid voltage when the plate voltage is

* Received by the Editor, May 9, 1918.

${ }^{1}$ I. Langmuir, "Proc. Inst. Radio Engrs.," 3; page 261, 1915. 
constant, the second, the variation of plate current with plate voltage with the grid voltage held constant. These characteristic curves are usually obtained by varying in steps the battery voltages applied between the elements of the tube and reading the plate current corresponding to the applied voltages. The slopes of these curves, being the ratio of current to voltage, have the dimensions of a conductance. This method is very slow and inaccurate.

In many cases the operation of a tube takes place about a point in the characteristic surface where the surface is nearly plane. Also when the variations are confined to a small area about the operating point and we are not concerned with distortion, the characteristic surface may be considered to be a plane. Thus the equation of the characteristic surface about an operating point $\left(i_{p}, v_{g}, v_{p}\right)$ may be written, as given by Vallauri ${ }^{2}$ in the form

$$
i_{p}=a v_{g}+b v_{p}+c
$$

where $a$ is the slope of the plate-current grid-voltage characteristic curve passing thru the operating point while $b$ is similarly the slope of the plate-current plate-voltage characteristic. The quantities $a$ and $b$ are fundamental in determining the behavior of a tube as an amplifier and oscillator as has been shown by Vallauri.

\section{Amplification Constant and Internal Resistance}

It is, however, more convenient to deal with the quantities $\frac{a}{b}$ and $\frac{1}{b}$. The first of these is the same as $k$ in Langmuir's equation; and, as mentioned before, is a constant for a given tube. It is called by $\mathrm{H}$. J. van der Bijl ${ }^{3}$ the amplification constant. The quantity $\frac{1}{b}$ is the internal a. c. resistance of the tube in the plate circuit and will be designated $R_{i}$. Its value depends upon the plate and grid voltages and to some extent upon filament temperature.

Assume that the plate circuit is closed thru an impedance $Z$, which may be the impedance of a pair of telephones in the case of ordinary use or may take the form of a pure resistance in the case of a resistance-coupled amplifier or the primary of a transformer in the case of a transformer-coupled amplifier.

${ }^{2}$ G. Vallauri; "L'Elettrotecnica," IV, 3, page 43, 1917.

${ }^{3}$ Unpublished paper of September 17, 1917. 
Assume also that an alternating e. m.f. $e_{g}$ is impressed between the grid and filament. It then follows that the alternating current in the plate circuit will be the same as that which would flow in a simple a.c. circuit in which the impressed e. m. f. is $k e g$ and which contains a resistance $R_{i}$ in series with the inserted impedance. ${ }^{4}$

To prove this theorem let us introduce the additional notation

$I p=$ d. c. component of the plate current.

$i=$ a. c. component of the plate current.

$E_{g}=$ voltage of grid battery.

$e_{g}=$ a. c. impressed e. m. f. on grid.

$E_{p}=$ voltage of plate battery.

and assume an impedance $z=(x+j y)$ inserted in the plate circuit which has a d. c. resistance $x^{\prime}$.

Then $i_{p}=I p+i$

$$
\begin{aligned}
& v_{g}=E_{g}+e_{\eta} \\
& v_{p}=E_{p}-I_{p} x^{\prime}-i(x+j y) .
\end{aligned}
$$

By Vallauri's equation we have

$$
i_{p}=a v_{g}+b v_{p}+c
$$

and substituting in this the values of $i_{p}, v_{g}$, and $v_{p}$,

$$
I_{p}+i=a\left(E_{o}+e_{g}\right)+b\left[E_{p}-I_{p} x^{\prime}-i(x+j y)\right]+c .
$$

But the steady current

$$
I_{p}=a E_{g}+b\left(E_{p}-I_{p} x^{\prime}\right)+c,
$$

hence

$$
\begin{aligned}
& i=a e_{g}-b i(x+j y) \\
& \frac{a}{b} e_{g}=i \frac{1}{b}+i(x+j y)
\end{aligned}
$$

or

$$
k e_{g}=i R_{i}+i(x+j y)
$$

This latter is the equation of e.m.f.'s for an impressed e.m.f $k e_{g}$ in a circuit with a resistance $R_{i}$ in series with an impedance $(x+j y)$.

As an example take the case of a resistance-coupled amplifier, in which case an alternating e.m.f. $e_{g}$ is impressed between the grid and filament of one tube and the e. m. f. across a resistance $R$ in the plate circuit of that tube is introduced into the grid circuit of the next tube. The voltage amplification is the

${ }^{4}$ The author is indebted to Mr. H. H. Beltz of the Bureau of Standards for suggesting this theorem. 
ratio of the e. m. f. handed on to the next tube, $i R$, to the impressed e. m. f., $e_{o}$.

We have

hence

$$
k e_{g}=i R_{i}+i R,
$$

$$
\frac{i R}{e_{g}}=k \frac{R}{R_{i}+R}
$$

This shows that the voltage amplification increases as the coupling resistance $R$ is increased, approaching as a maximum the value of the amplification constant $k$. This assumes that $R_{i}$ and, therefore, the actual voltage between plate and filament, remains unchanged. Other amplifier problems and the conditions for oscillation of numerous circuits may be readily worked out by the use of this simplifying theorem.

Experimental Method for Determining the Amplification Constant and Internal A.C. Resistance of Three-Electrode Vacuum Tubes

Instead of deriving the values of the amplification constants and internal resistance indirectly from the static characteristic curves, they may be determined directly and rapidly in a very simple manner using alternating current of audio frequency. The circuit is that shown in Figure 1, in which $c d$ is a slide wire supplied with a small audio frequency current from an alternator thru a step-down transformer or by coupling to a tube source. A variable ground connection not shown in the figure is likewise put on a rheostat which is in parallel with the slide wire in order to obtain a better minimum in the phones. $R$ is a dial resistance box going up to about ten thousand ohms which may be connected in circuit by means of the switch $S$.

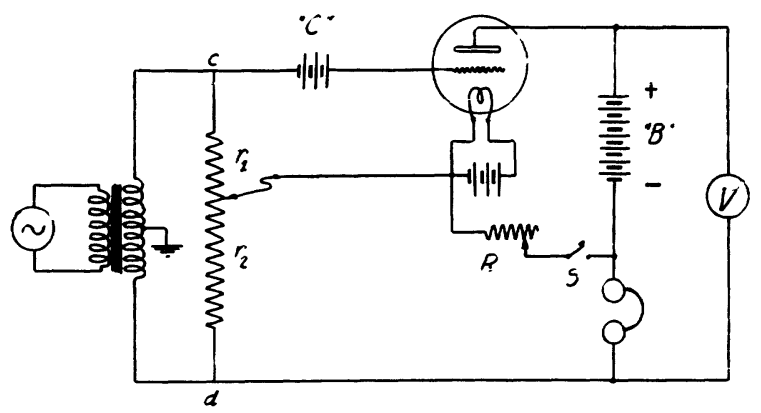

FIgURE 1 
First, the amplification constant $k$ is determined with the switch $S$ open, by adjusting the slider until the telephones are silent when

$$
k=\frac{r_{2}}{r_{1}} .
$$

To determine the internal resistance $R_{i}$, the switch $S$ is then closed and the slider set at, say, the middle point and silence is again obtained by varying the resistance $R$. Then

$$
R_{i}=(k-1) R
$$

Any other definite ratio of $\frac{r_{2}}{r_{1}}$ (less than $k$ ) can be used in which
case

$$
R_{i}=\left(\frac{r_{1}}{r_{2}} k_{i}-1\right) R
$$

The above expressions are readily proven. In the first determination an e. m. f. $e_{g}$ which is proportional to $r_{1}$ is introduced between the grid and filament. As pointed out before, this has the effect of impressing an e. m. f. $k e_{\theta}$ in the plate circuit. The e. m. f. across $r_{2}$ which is $\frac{r_{2}}{r_{1}} e_{g}$ is $180^{\circ}$ out of phase with the e. m. f. $k e_{g}$ and will balance it when $\frac{r_{2}}{r_{1}} e_{g}=k e_{g}$. Hence

$$
k=\frac{r_{2}}{r_{1}}
$$

In the second case an e. m. f. $e_{g}$ proportional to $r_{i}$ is likewise impressed between grid and filament, which will produce an alternating current $\frac{k e_{g}}{R_{i}+} \bar{R}$ in the plate circuit provided no current flows thru the phones. The e.m.f. across the resistance $R$ is $\frac{k e_{g} R}{R_{i}+R}$. This is balanced by the e. m. f. $\frac{r_{2}}{r_{1}} e_{g}$ across $r_{2}$ when

$$
\frac{r_{2}}{r_{1}} e_{g}=\frac{k e_{g} R}{R_{i}+R} \text { or } R_{i}=\left(\frac{r_{1}}{r_{2}} k-1\right) R,
$$

and in the case when the slider is set at the middle point $\left(r_{1}=r_{2}\right)$,

$$
R_{i}=(k-1) R \text {. }
$$

As noted before, the amplification constant is very nearly a constant for a given tube, but the internal resistance $R_{i}$ varies with plate and grid voltages and to some extent with filament current. Curves may be obtained for $R_{i}$ as a function of these variables obtaining directly and accurately all of the data furnished indirectly and inaccurately by the static characteristic curves excepting the grid current characteristics. 
In the actual set-up, the slider $\mathrm{c} d$ of Figure 1 was seven ohms in resistance and consisted of ten turns of resistance wire inductively wound on a marble cylinder, each turn corresponding to one hundred divisions on the scale. It would be preferable to use a straight wire in order to reduce the inductance. This can be marked to read amplification constant directly. The current in the slide wire and hence the voltages acting on the tube should be kept so small that the operation of the tube takes place over a portion of the characteristic so limited that it is practically a straight line. In the measurements described herein, a current of 50 milliamperes or less was used. This current was supplied by either an alternator or tube source and up to 2,000 cycles per second, and for the tubes investigated no change due to frequency was observed. A buzzer source of interrupted current will suffice in many cases.

The dial resistance box $R$ consisted of non-inductively wound coils and, as noted before, went up to ten thousand ohms. In some cases the internal resistance of the tube may be so high that it becomes necessary to use a ratio of $\frac{r_{1}}{r_{2}}$ greater than unity in order that a balance can be obtained with this box.

In obtaining values of the internal resistance for given plate voltages, it must be remembered that since the direct current flowing thru the tube must flow thru the telephones and $R$ in parallel, the actual voltage applied to the tube will be somewhat less than that of the plate battery. In order to measure the actual voltage on the tube, the voltmeter should be connected across the battery and telephone receivers as shown in Figure 1, and the measurement made with the voltmeter key closed. It is desirable to use a high resistance voltmeter and telephones of moderately low resistance, since then the applied voltage when the measurement of $k$ is made, with the switch $S$ open, will differ only slightly from that acting when $R_{i}$ is measured.

In Figures 2 and 3 are shown curves of the internal resistance with varying plate voltages for two types of tubes, each intended for use as amplifiers or detectors. Tube 1 has an amplification constant of 14.5 while Tube 2 has only 7.5. The resistance of the former is, however, very much greater than that of the latter even when the plate voltages are, respectively, 100 and 20 . This shows that, for a given type of tube, the associated apparatus should be designed so as to fit the tube characteristics or, with given apparatus, a tube should be chosen which most nearly fits the apparatus. 


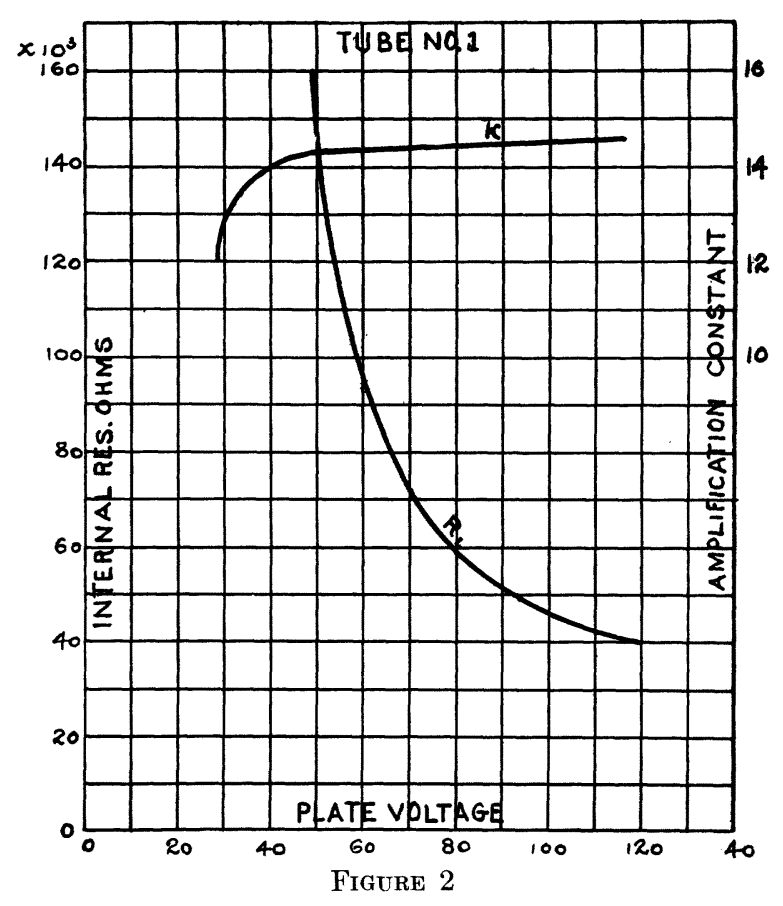

The above method has been applied for audio frequencies and to tubes of sufficiently high vacuum and operated at such

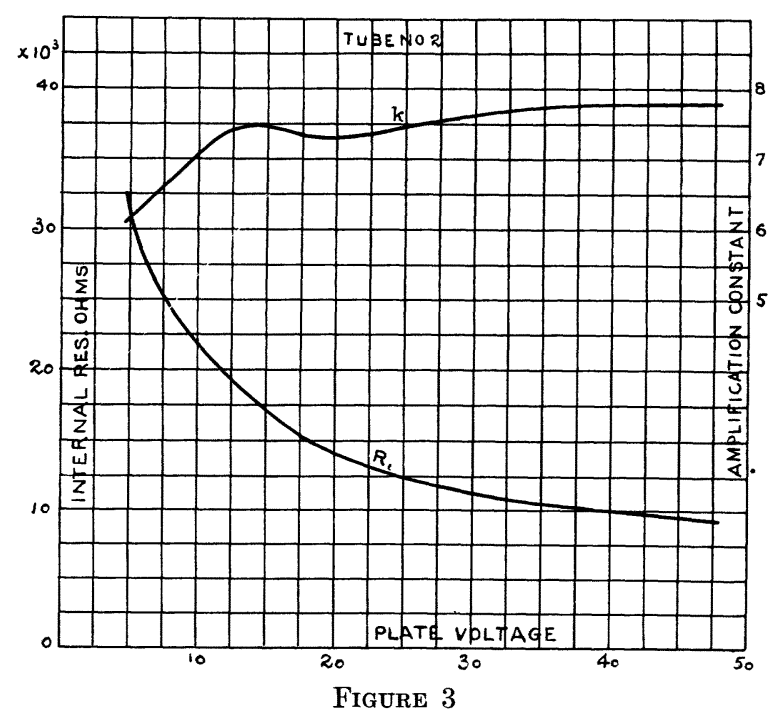


voltages that there was no lag of the plate current with respect to the applied grid and plate voltages. The method can, without doubt, be modified so as to measure the phase angle of the lag if such exists and to determine the dynamic values of the tube constants at radio frequencies.

$$
\begin{array}{r}
\text { Washington, D. C. } \\
\text { May } 3,1918 .
\end{array}
$$

SUMMARY: After considering the characteristics and "amplification constants" of three-electrode vacuum tubes, the author describes the theory and practice of a new method for determining the amplification constant and internal resistance of such tubes directly and rapidly. Examples of results thus obtained are given. 